Author(s): Hyyti, Heikki \& Visala, Arto

Title: $\quad$ Feature Based Modeling and Mapping of Tree Trunks and Natural Terrain Using 3D Laser Scanner Measurement System

Year: 2013

Version: Post print

Please cite the original version:

Hyyti, Heikki \& Visala, Arto. 2013. Feature Based Modeling and Mapping of Tree Trunks and Natural Terrain Using 3D Laser Scanner Measurement System. 8th IFAC

Symposium on Intelligent Autonomous Vehicles, Australia, 2013. Intelligent Autonomous Vehicles. Volume 8, Part 1. P. 248-255. DOI: 10.3182/20130626-3-AU-2035.00065.

All material supplied via Aaltodoc is protected by copyright and other intellectual property rights, and duplication or sale of all or part of any of the repository collections is not permitted, except that material may be duplicated by you for your research use or educational purposes in electronic or print form. You must obtain permission for any other use. Electronic or print copies may not be offered, whether for sale or otherwise to anyone who is not an authorised user. 


\title{
Feature Based Modeling and Mapping of Tree Trunks and Natural Terrain Using 3D Laser Scanner Measurement System Heikki Hyyti*, Arto Visala*
}

\author{
* Department of Automation and Systems Technology, Aalto \\ University School of Electrical Engineering, P.O. Box 15500, \\ 00076 Aalto, Finland (e-mail: firstname.lastname@aalto.fi)
}

\begin{abstract}
This paper presents a novel approach to measure tree trunks and to model the ground using a 3D laser scanner. The 3D scanner, self-build using two 2D Sick scanners on a rotating base, measures each scan line approximately at $45^{\circ}$ angle towards the ground and the trees. Single scan lines are segmented to find ground and tree returns. 3D point clouds from the surrounding forest are recorded while the measuring vehicle is moving. Sequential scan lines are joined together as the pose changes are reduced from the older buffered measurements. Laser odometry and inertial measurements are used to measure the pose changes. The ground is modeled by fitting a $1 \mathrm{~m}$ grid to $3 \mathrm{D}$ point cloud extracted using a ground return detector. Tree trunks are searched from the 3D point cloud using a histogram approach to segment measurements into separate point clouds for each tree trunk. Tree trunks are modeled using ten circle features one on the other using the extracted point cloud. Instead of using the whole point cloud, mapping is done only for the extracted features and the travelled path to save computation time. Our method can detect nearly all tree trunks and measure them on short ranges of less than $8 \mathrm{~m}$ with errors less than $4 \mathrm{~cm}$ in diameter.
\end{abstract}

\section{INTRODUCTION}

Autonomous navigation and manipulation in unstructured environments is a major challenge in robotics. In order to work efficiently in the environment, the robotic system needs detailed information about the surrounding terrain. It is crucial to be able to avoid obstacles and detect impassable terrain through foliage and vegetation. In many dense environments and in the northern regions, it is usually not reasonable to rely only on satellite navigation. GPS satellites are on quite low orbits and therefore, especially in northern regions, tree canopy is usually blocking the signal. It is therefore helpful to use optical and laser sensors to detect and map the environment. In our case, the studied environment is Finnish pine forest shown in Fig 1.

In our study the aim is, in addition to navigation and manipulation in forests, to accurately measure and model the trees for the forest industry. The measurement system should work autonomously without intervening in the work of forest machines. There is a need for reliable continuous tree detection, measurement and mapping system, which uses reliably low cost sensors and can measure while the forest machine is operated. Usually in robotic applications, the forest is perceived using a tilted or rotated laser scanner, i.e. LiDAR, or a commercial three-dimensional laser scanner.

In the work by McDaniel, Nishihata, and Brooks (2012) the laser scanner was tilted in place to gather $3 \mathrm{D}$ data in forest environment. In the work by Lalonde et al. (2006) the laser scanner was rotated and in study by Henning and Radtke (2006) and similarly in the thesis by Forsman (2001) they used a Riegl 3D laser scanner to gather data. They all have in common that they first gather a 3D data set stationary and then analyze it as a 3D point cloud. Instead, we use two rotated 2D scanners and analyze and segment the data from individual scan lines while moving. There is practically no time delay between measurements on a same scan and therefore there is only minor errors caused by pose changes or other movement in the environment. Therefore, it is advantageous to segment data using only single scan lines. After the segmentation, all data is joined to a $3 \mathrm{D}$ point cloud and analyzed as a whole. The result of segmentation is then added for every measurement as extra information for later processing.

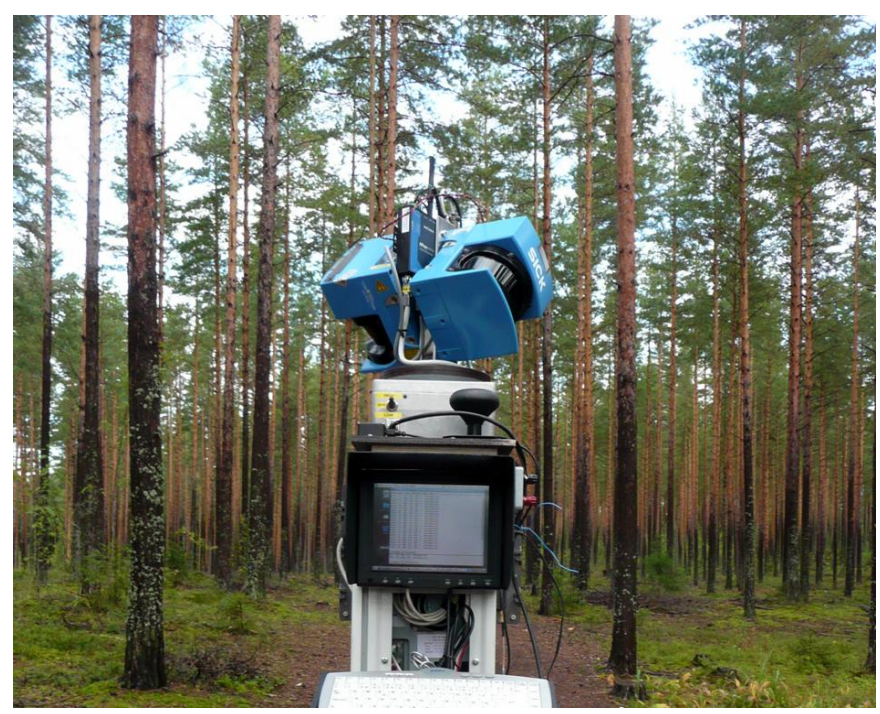

Fig. 1. Measured pine forest and the rotating 3D laser scanner on an all-terrain vehicle driving through the forest while measuring. 
In our study we use two $45^{\circ}$ rotated Sick LMS-200 scanners mounted back to back on a rotating base built in our laboratory (see Fig. 1). Because of the system setup, we can identify ground measurements and tree trunks from individual $45^{\circ}$ rotated scan lines. Therefore we can divide our 3D laser scanner data to ground and non-ground returns using the algorithms presented in this paper. Similarly we can identify tree edges from individual scans using quite simple edge detectors. This paper presents methods for dividing 3D point cloud to different tree trunks and measuring trunk diameters at different heights. Tree trunks are modeled as ten circle features (locations and radii) on top of each other at heights from ground to $10 \mathrm{~m}$.

The three-dimensional forest mapping problem can be reduced into a two-dimensional problem using terrain model and tree trunk features. The terrain model is used as a reference height for tree trunk features. This is an efficient way to reduce the $3 \mathrm{D}$ mapping problem to two dimensions and to simultaneously get diameter measurements from tree trunks at different heights.

The presented method is built on a laser odometry method by Hyyti et al. (2009). It is assisted with an inertial measurement unit (IMU). The error of the estimated pose of the measurement system is kept small enough to gather measurements simultaneously as the measuring vehicle moves on a rough forest floor. Using our method, the robot or measuring vehicle does not need to remain stationary during data collection as in the referred work (McDaniel et al. 2012, Henning, Radtke 2006, Lalonde 2006).

As the mapping is only done for the extracted features, the amount of mapped data is quite small. In comparison large point clouds are usually used to build a map in multidimensional SLAM cases, for example in the 6D SLAM article by Nüchter et al. (2007). In our work there is no need to handle whole point cloud as only extracted features are used in the mapping process. This significantly reduces memory and processing requirements.

\section{MEASUREMENT SYSTEM AND ENVIRONMENT}

The measurement system consists of a Sick LMS221 laser scanner, inertial measurement unit (IMU), GPS and a selfbuilt 3D scanner. The 3D laser scanner was built by using two Sick LMS200 scanners, which were tilted $45^{\circ}$ sideways on a rotating base (see Fig. 1). Alignment of the Honda allterrain vehicle was measured using a Microstrain Inertia-Link inertial measurement unit (IMU). A generic GPS-receiver from a Ponsse forest harvester was used to gain a global position reference for the built map. Unfortunately GPS-receiver was not accurate enough to be used as a reference measurement for accuracy of mapping. The self-built 3D laser scanner system shown in Fig. 1 is rotated at approximately 30 rpm. It thus takes about a second to gather a data set that covers the full field of view of $360^{\circ}$ around the system.

The Sick LMS221, which was mounted horizontally in front of the Honda all-terrain vehicle, is used to measure the pose change using the laser odometry method (Hyyti et al. 2009) and IMU. The estimated pose changes are compensated from the $3 \mathrm{D}$ point cloud so that the device can move while measuring.

The measurements were taken in a forest by Finnish Forest Research Institute, Metla. The forest was growing mainly sparse pine tree as seen in Fig. 1, but there were denser areas with pines, spruces and some deciduous trees around. The terrain around the measuring region was diverse, with flat and hilly regions. According to the Finnish Forest Research Institute, the four most significant tree species in Finland are Scots pine (Pinus sylvestris) 65.5\%, Norway spruce (Picea abies) $23.7 \%$, Downy birch (Betula pubescens) $6.1 \%$, and Silver birch (Betula pendula) 2.7\% (Metla 2007).

\section{MEASUREMENTS AND POSE COMPENSATION}

The data was collected using a self-built 3D scanner shown in Fig. 1. One laser scanner measurement consists of 181 range measurements i.e. returns at one degree resolution. This twodimensional measurement data i.e. scan line is transformed to three-dimensional global coordinates according to the measured rotation of the base. The scan line is then transformed using the pose change of the vehicle which is estimated using a combination of the laser odometry method (Hyyti et al. 2009) and IMU. In the used method a horizontal laser scanner data set is correlated with a previous data set. The horizontal translations are calculated using the laser odometry method and the vertical translation is neglected as the map is built in $2 \mathrm{D}$ using $3 \mathrm{D}$ features. The three-axis rotation is measured using the IMU. The drifting bias of a yaw angle is estimated in the SLAM-process using map and inertial information together. The whole sensor fusion and mapping process is documented in the Master's Thesis by Hyyti (2009).

To be able to recognize tree trunks and to model the ground using 3D measurements, a lot of scan lines have to be attached together. This is done by buffering collected and transformed laser scanner returns in a measurement buffer. Old values in the buffer are translated and rotated using the estimated pose change of the vehicle. Then, new returns are added to the end of the buffer. The buffered point cloud is thus transformed according to the current pose of the vehicle. The buffer length is determined to half revolution of the scanner giving full coverage of the environment. All the returns were indexed to ground, tree or other returns using ground detector (see Section 4) and tree trunk detector (see Section 5). This segmentation is done for a single scan line before adding the measurement into the buffer.

Pose estimation errors accumulate in the measured 3D point cloud. The longer a 3D data set is taken, the more errors are cumulated to the data. Therefore, the buffer should be as short as possible to get an accurate point cloud. Still, there needs to be enough information about environment to detect ground and tree trunks. To get a full $360^{\circ}$ data set around the scanner, a measurement of at least a second duration is needed. An example of a pose corrected and buffered point cloud is shown in Fig. 2. This data is used to search tree trunks and model the ground level. The buffer is essential for tree trunk search and modeling, because tree features have to be searched from a full point cloud to avoid erroneous detections or inaccurate tree location and diameter measurements. 


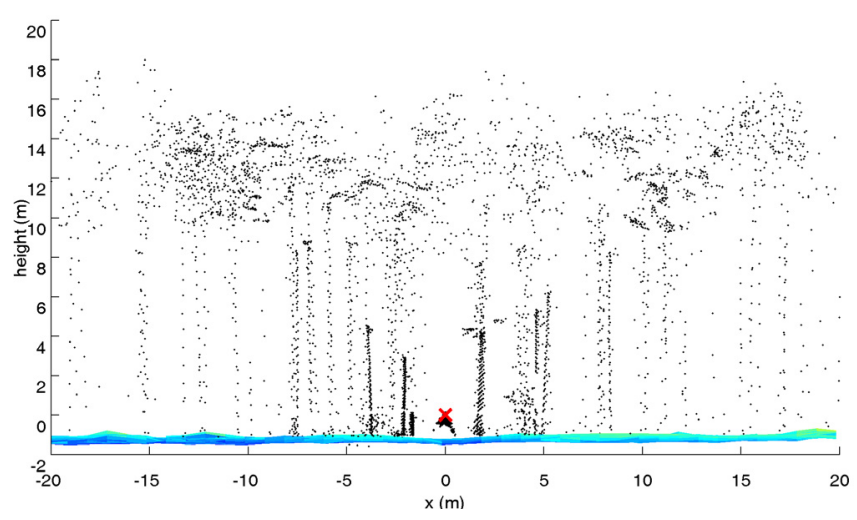

Fig. 2. A set of 3D laser scanner measurements viewed from side. The measuring vehicle is plotted with a red cross. A terrain model is drawn instead of ground returns.

Errors in the estimated pose change during a time of a second proved to be small compared to measuring errors mainly caused by inaccuracies in the angle measurements of the selfbuilt rotating base and inaccuracies in the data of Sick LMS200 laser scanner. The most reliable measurements were got near the measuring vehicle as there were angular errors in the point cloud. We are currently building a new prototype of the rotating $3 \mathrm{D}$ laser scanner to get more accurate measurement for the rotating base and for the pose of the 3D scanner.

\section{TERRAIN MODELING}

In ground segmenting and terrain modeling phase, every laser scan line is first segmented for ground returns. Both of the scanners are fixed to the rotating base in 45 degree angle and thus every individual scan line contains a part of returns from the ground and other part from obstacles upper. The ground is usually seen as linearly arranged points in the scan plane at least when the ground or measuring vehicle are a lot less than 45 degrees inclined. The vehicle or forest harvesters can not move in such a deep slopes, so it can safely be assumed that slope of the terrain and vehicle inclination are lot less than 45 degrees.

The ground level is found from a single laser scan by iteratively fitting a line to the measured returns at the lower part of the data (on the left side in Fig. 3). Returns that are near the fitted line or on the left side of the line are marked as ground returns. The algorithm assumes that the ground is not seen through and is nearly linear. The position of the fitted ground line is initially selected to be parallel to y-axis and go trough the leftmost returns in the selected window that is shown in the right part of Fig. 3. Then the returns that are near enough the fitted line are selected as ground returns to where the line is refitted. This process is iterated a few times using decreasing margins to get a sufficiently reliable estimate of the ground returns.

The quality of the fitted line is estimated as the amount of ground returns (orange points in Fig. 3). Because ground returns are fitted to points with only small distance to line, there are enough returns only when there is a flat area. If the number of ground returns is smaller than adjusted limit, the ground fitting is assumed to be incorrect and no ground re- turns are found from the current scan. Other line fitting methods, for example iterative closest point (ICP) should work as well, but the heuristic method with only a few iterations was used to save computation time.
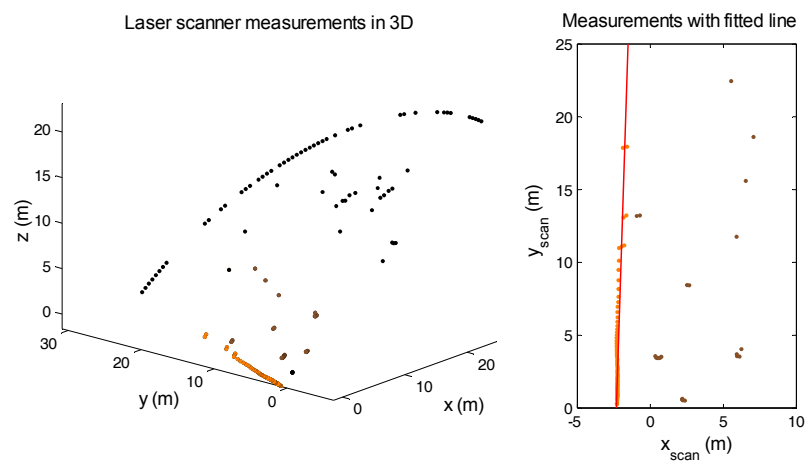

Fig. 3. Ground return detection for single scan data. The red line is the resulting fitted line to orange points representing detected ground returns.

All data in the data buffer is segmented according to their detection in different detectors. There is a lot more ground returns returned from nearer the measuring vehicle than from further away. The distribution of ground returns is flattened by randomly dropping nearer returns out using empirically fitted probability function of detected ground returns as a function of range (Hyyti 2009). The size of the point cloud is reduced to satisfy memory and processing time requirements. The filter leaves enough equally distributed ground returns to the ground buffer so that the terrain model can be efficiently fitted to the remaining point cloud.

The terrain model is generated from the remaining equally distributed ground returns in the buffer. These points are fitted in $1 \mathrm{~m}$ grid, where the height is estimated by the median of the points around every grid cell. $1 \mathrm{~m}$ grid is used, because it is accurate enough to be used in the remaining tree modeling to remove the effect of differences in ground height and it still hided small variations in the ground and it was robust against errors in the segmentation of ground returns. The ground returns after the statistical drop out process and the terrain model are depicted in Fig. 4. In the figure the terrain model is colored by the height of the model.

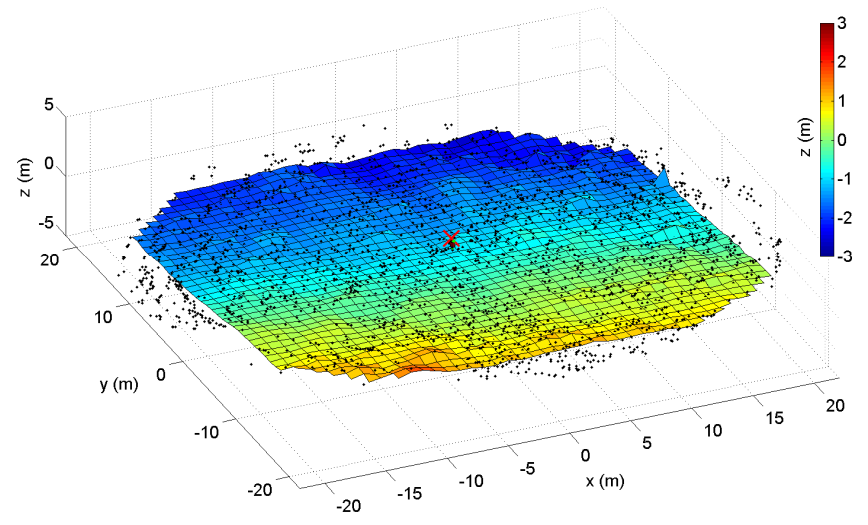

Fig 4. The terrain model using $1 \mathrm{~m}$ grid with ground returns. The measuring vehicle is located at a red cross. 


\section{TREE TRUNK SEARCH AND MODELING}

The search for trees is done partly from individual laser scans and partly from the buffered measurement set in 3D. Otherthan-ground returns are segmented using a tree edge detector. It is used to label the returns to left and right edges of tree trunks, tree returns in the middle and other returns. The edge detection is done in the $45^{\circ}$ tilted $2 \mathrm{D}$ scanner coordinates using only data of a single scan line. As tree trunks are nearly vertical and a scan is $45^{\circ}$ orientated, the tree is seen approximately in $45^{\circ}$ angle. The used detector is a process that searches for large differences between adjacent scanned angles and detects left and right edges of a tree trunk, as well as the tree returns between the detected edges. Similar detectors are used in many 2D mapping cases by many authors (Guivant, Nebot 2001, Jutila, Kannas \& Visala 2007, Bailey, Nebot 2001).

In previous work (McDaniel et al. 2012, Lalonde 2006, Henning, Radtke 2006, Aschoff, Thies \& Spiecker 2004), the tree search is done by grouping and segmenting laser scanner returns in 3D coordinates. In contrast, in our work, the complex tree search and point cloud grouping operations are simplified by first detecting both tree edges and the tree returns between them to remove other noisy measurements from point cloud. In addition, the detected ground returns are left out of the trunk detection process.

Tree trunks are searched from the point cloud by selecting only labeled tree edge and tree returns. A $2 \mathrm{D}$ histogram is fitted to this $3 \mathrm{D}$ point cloud using a bin width of $0.2 \mathrm{~m}$ in both $\mathrm{x}$ and $\mathrm{y}$ directions. The trunks are supposed to be nearly vertical so they should be seen as local maximums in the $2 \mathrm{D}$ histogram. The bin width was selected to be so small $(0.2 \mathrm{~m})$ that no two different trunks can be fitted inside the same bin. The number of returns inside one histogram bin is highly biased by the measuring range. To separate non-tree measurements from the real trees, a threshold value is used to classify histogram bins as trees (or non-trees).

The threshold is determined using a model of a stereotypic tree trunk as a function of the measuring range. For simplicity in the model, the number of returns is assumed to be equal at every steradian in the FOV of the 3D scanner. A solid angle, in which the model trunk is seen, is estimated as a function of measuring range. The model is depicted in Fig. 5 from side on left side and from top on the right side of the figure.

The visible height of a tree trunk depends on the measuring range. If the tree is far enough, the height is cropped to 10 meters $\left(Z_{\max }\right.$ in Fig. 5). The tree modeling is only done from ground to $10 \mathrm{~m}$ height and it was therefore reasonable to drop out returns higher than $10 \mathrm{~m}$ from ground. At the first region (yellow) in Fig. 5, only the field of view of the 3D scanner is limiting the number of returns from the model trunk. At the second region (green) the ground is also limiting measurements, and at the third one (cyan) also the upper limit of $Z_{\max }$ limits the number of possible returns. Different regions are depicted in Fig. 5.

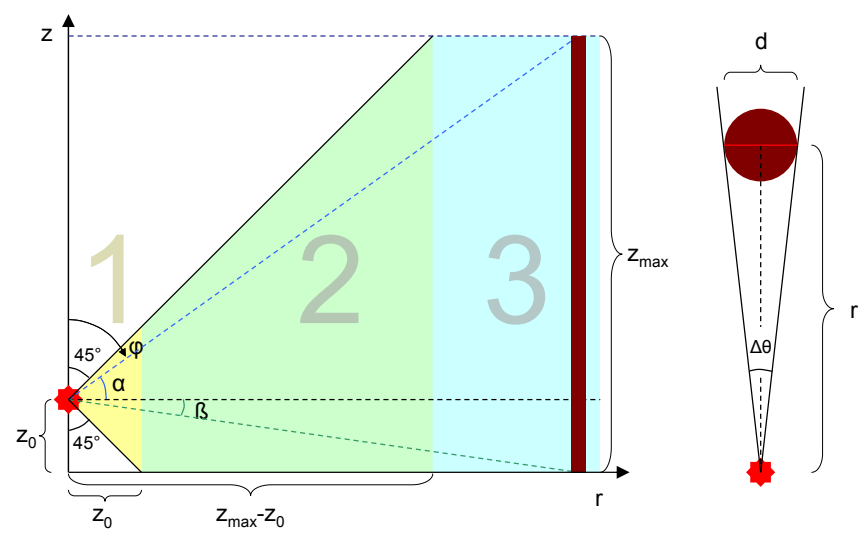

Fig. 5. Estimation of the number of points returned from a single tree trunk. The trunk model is viewed from side and top. At the region 1 (yellow) the FOV is not restricted, at region 2 (green) the FOV is limited by ground and at region 3 (cyan) $Z_{\max }$ limits the FOV.

The mathematical model is presented in Equation (1), where $\Omega$ is a solid angle, $\varphi$ is a polar angle, $\theta$ is an azimuthal angle, $r$ is the tree range, and $d$ is a diameter of the model trunk. Ranges $Z_{0}$ and $Z_{\max }$ are the limiting constants depending on the sensor assembly and the used data. The $45^{\circ}$ angle shown in Fig. 5 or in Equation (1) is a parameter caused by the construction of the laser scanner. The visible solid angle of the model tree trunk is thereby:

$$
\begin{aligned}
& \Omega=2 \arctan \frac{d}{2 r}(\sin \alpha+\sin \beta) \\
& \alpha=\min \left[\frac{\pi}{4}, \arctan \left(\frac{z_{\max }-z_{0}}{r}\right)\right] \\
& \beta=\min \left[\frac{\pi}{4}, \arctan \left(\frac{z_{0}}{r}\right)\right]
\end{aligned}
$$

The number of returns in a histogram bin and the modeled limit is shown in Fig. 6. The calculated threshold model is used in Fig. 7 to suppress returns from bushes and other smaller-than-tree objects. The same trunks that are shown in Fig. 6 are shown in the 2D histogram in Fig. 7. The plus sign at the centre of the image is the current location of the measurement system. As it can be seen in the 2D histogram, only densely packed groups are detected as tree trunks.

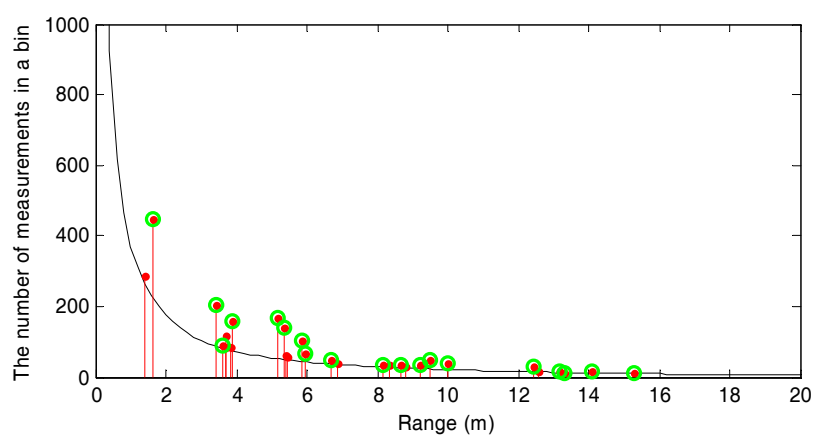

Fig. 6. Trunk detections as a function of the measuring range together with the threshold function. Red lines are trunk candidates and the green circles are accepted tree trunk locations. The range is measured from the origin in Fig. 7. 


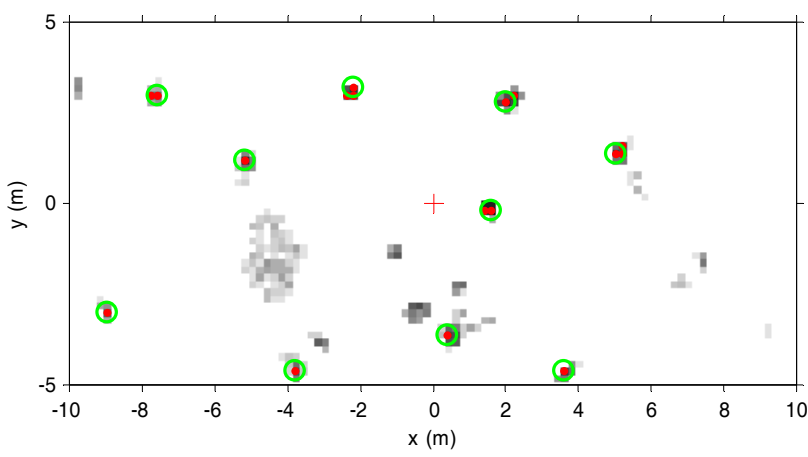

Fig. 7. A part of the 2D histogram. The red points are tree trunk candidates and the green circles are accepted tree trunk locations. A red plus is the origin where the range in Fig. 6 is measured.

The approximate trunk locations (green circles in Fig. 7) are found from the 2D histogram using a local maximum around every candidate (red dots) which exceeded the modeled threshold function (black curve). Measurements nearer each other than $1 \mathrm{~m}$ are supposed to belong to the same trunk. This allows only the largest histogram bin around a same trunk (green circles in Fig. 7) to be used in the tree trunk modeling phase.

Tree trunk modeling is done for every tree candidate from the previous 2D histogram. All measurements in the buffer except ground returns around a tree candidate are taken and a 3D line is iteratively fitted to the data set discarding the furthest points out as outliers (see Fig. 8). Line fitting is used for approximately finding the orientation and location of the tree trunk. The amount of outliers is approximated with a distance histogram to the fitted line (Hyyti 2009). The first small enough histogram distance is detected as first location where trunk returns end and most of returns are from foliage or branches. There are often quite large amount of outliers in the data, but distance histogram can usually classify trunk measurements from foliage. In Fig. 8 the red line is the fitted line and black dots are the accepted measurements using the distance histogram. Magenta dots are detected as outliers. a)

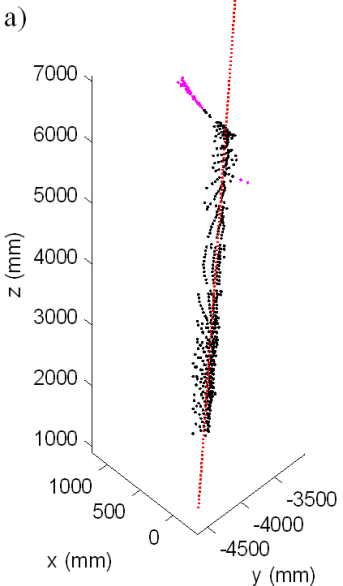

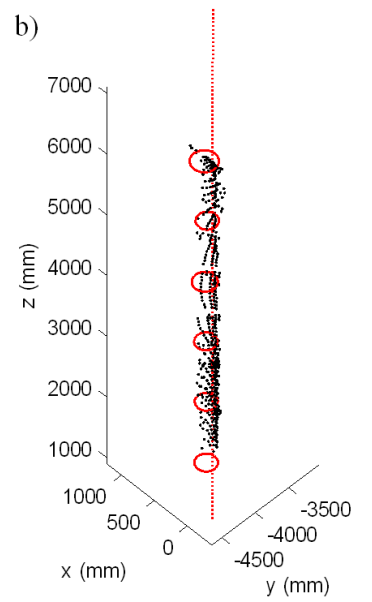

Fig. 8. Data filtering, reorientation and tree trunk modeling. The black dots are accepted measurements where the red circles are fitted. Magenta dots are detected as outliers.
The line fitting is done using least squares line fitting technique (Weisstein 2012) for xz- and yz-planes separately. The error in the fitting procedure is calculated against $\mathrm{z}$-axis, because tree trunks are usually aligned vertically. The fitted line and the found outliers are shown in Fig. 8a. In the second phase, the segmented data is reoriented according to the fitted line as shown in Fig. 8b. Next the reoriented data is split into a ten overlapping data sets at every height from 1 to $10 \mathrm{~m}$ on top of each other. The data regions overlap by half a meter in both directions as the circle fitting phase needs as much data as possible.

At every height where there are enough data points to fit a circle, a closed form circle fitting procedure presented by Ian Coope (1993) is used to fit circle to measurements. It is used iteratively to minimize the effect of outliers. Fig. 9 shows one iteration step. The dashed circle is an initial fitted circle. The orange area around it is a safe region. Width of the safe region is the variance of the distances from measurements to the fitted circle. One standard deviation is used to detect outliers from the circle fitting process. The fitted circle without detected outliers is shown in Fig. 9 with solid red line. Found outliers are marked with magenta dots in the figure.

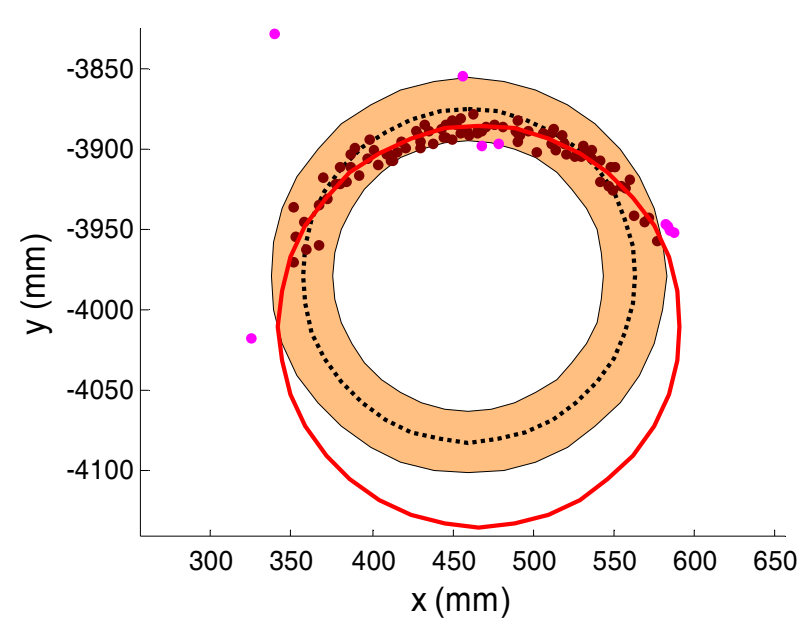

Fig. 9. The iterative circle fitting method. Magenta dots are outliers and brown dots are accepted measurements at first iteration. The red circle is the refitted model.

The iteration of the algorithm was done three times to get a sufficiently accurate estimate of the circle. The number of needed iterations was determined empirically and after three iterations there were usually no outliers left in the fitted data. The location and the radius of the found circle are used as a circle feature in the mapping process. For example in the Fig. $8 \mathrm{~b}$ there are six successfully fitted circle features on top of each other. If any of these steps failed in the feature extraction, the circle feature was not used. If the detection of all circle features on the same tree trunk failed, the whole tree was not found in the fitting process. Therefore the map, which is mainly used to locate and measure tree trunks, can not be directly used in navigation or obstacle detection. Instead, the 2D histogram data (Fig. 7), which was used in the tree search, could be used in the navigation process to determine if there are obstacles on the way. 


\section{RESULTS}

The tree search and modeling was done for the buffered 3D point cloud once per second. Fig. 10 presents the tree search process in practice. Detected locations are drawn using a red circle under each found tree trunk location at ground level. All returns from the left and right edges using the tree detector are drawn in Fig. 10 using orange and black colored dots. Only the edge returns are plotted in Fig. 10 to reduce the amount of visible data and to demonstrate the quality of the low level data segmentation done using a single scan line. The figure shows that nearly all trees are found using the presented histogram tree search method. The method did not find any false trees, but some of the trees were not found in all iterations. Thus the extracted tree trunk features are quite reliable and can be used with mapping algorithms.

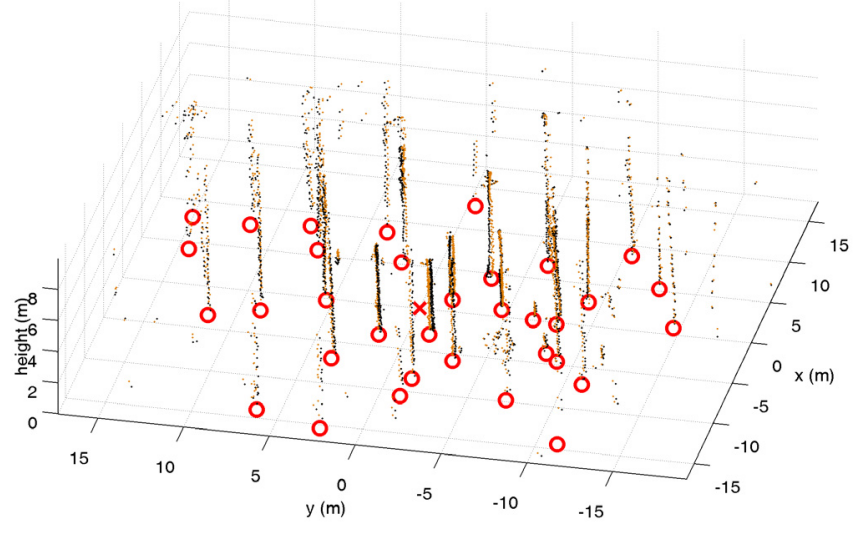

Fig. 10. The found tree trunks using 2D histogram method.

The estimated tree trunk locations are plotted at the ground level using red circles. Segmented returns from right edges are drawn using orange and left edges using black colored dots.

Diameters of the tree trunks at different heights are estimated using the circle fitting algorithm. When the range is smaller than $8 \mathrm{~m}$, nearly all diameters are measured with smaller error than $20 \mathrm{~mm}$ in radius $(40 \mathrm{~mm}$ in diameter). All measured radii (half of the diameter) are shown in Fig. 11 as a function of measuring range with the average and the standard deviations of the data. The figure is generated from all mapped circle features by using estimated feature as a reference. The median circle radius is reduced from all estimates at every tree height separately. Unfortunately, there were not accurate enough reference data available from the real locations or parameters of tree trunks. The reference measurement for tree diameter was measured using horizontal laser scanner measuring at an angular resolution of $0.25^{\circ}$ and a tree diameter estimation method by Jutila, Kannas \& Visala (2007). The reference has small uncertainty, which is plotted to Fig. 12 together with measurements of tree radii from the same tree (number 15 in Fig. 13 at the end of a driven path). The reference and measurements using 3D scanner are taken approximately around the same height between 1 and 2 meters. The result shows that there is an underestimate of approximately $2 \mathrm{~cm}$ in the radii $(4 \mathrm{~cm}$ in diameter) estimate of $3 \mathrm{D}$ scanner compared to the reference measurement.
The measurements shown in Fig. 11 are nearly normally distributed except for some erroneous measurements from ranges larger than $8 \mathrm{~m}$. The angular resolution of the scanner limits the measurement accuracy at larger ranges. At long range, there are only a few adjacent returns for the same tree trunk. While this limitation is taken into account in the mapping, the accuracy of trunk measurements can be increased by giving more value for measurements taken with sorter ranges.

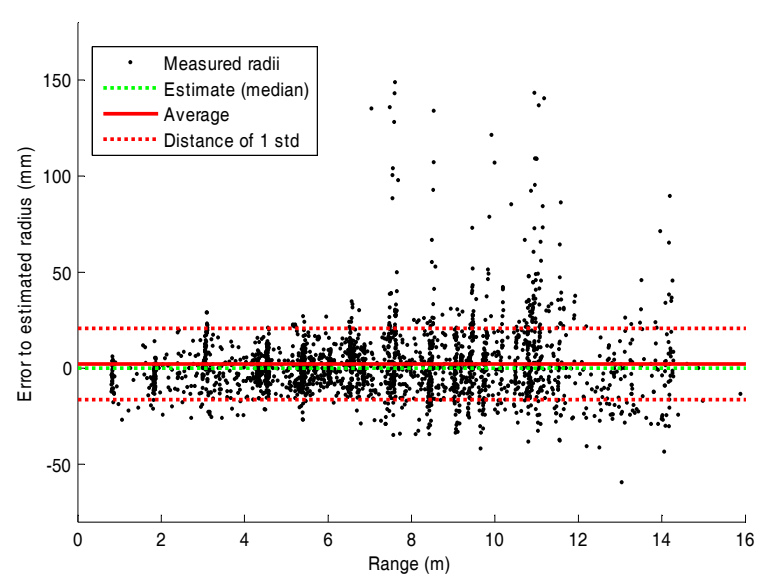

Fig. 11. Difference of radius compared to the median estimate for all circle features in the forest map as a function of the measuring range. Most of the data has small variations but there are some erroneous measurements with large radii at ranges larger than $8 \mathrm{~m}$.

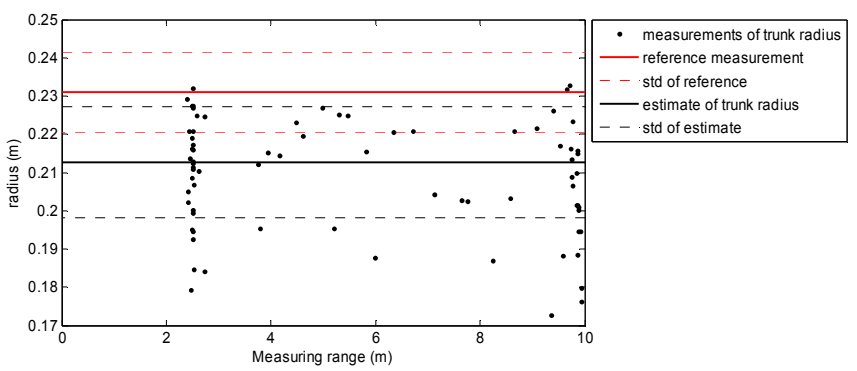

Fig. 12. Measured tree radii for a single tree at the average height of 1 to $2 \mathrm{~m}$ with reference measurement acquired using horizontal laser scanner. There is nearly $2 \mathrm{~cm}$ difference between reference measurement and the estimated tree radius. The estimate for trunk radius is computed using median of the data over all ranges.

Fig. 13 presents a 3D map of a small data set in the forest, as one tree (number 15) is approached and then vehicle is reversed back to the starting location. In the map, each tree trunk is modeled using at maximum ten circles on top of each other. Trunks in the map are plotted as a center line in the middle of circle features plotted with red color. The map is generated using medians of all of the gathered circle features at every height. Only those parts of trees are mapped that have had enough returns in the circle fitting process to minimize the amount of outliers. The map is generated using the mapping method by Hyyti (2009). 


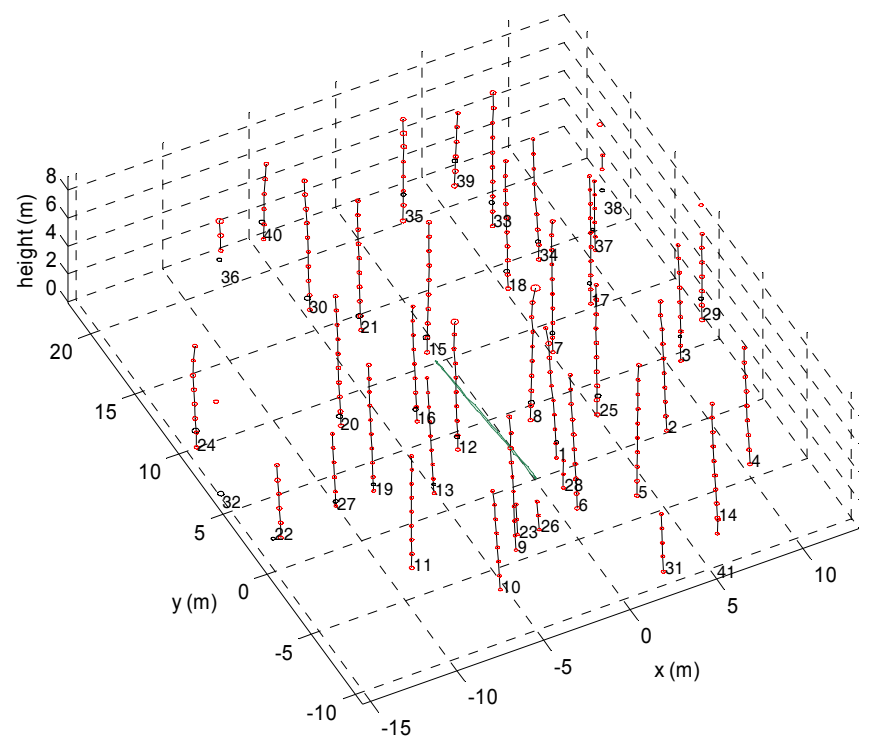

Fig. 13. A map of a small data set of a drive to tree 15 and back. Tree diameter measurements at different heights are plotted with red circles around a centre line of a tree drawn with black line. Green line shows the driven path.

\section{DISCUSSION}

The methods in this paper allow tree trunks to be used as features to map and measure forest environment. Most of the errors and inaccuracies in the current version of the work are caused by the inaccurate 3D point cloud measurement. Some errors are caused by the movement while measuring. The measuring system works in the forest, where there are pillar like pine trees with only a few low branches and undergrowth. The method has to be developed further in order to use it in denser spruce forest or with younger pine trees with more branches or with denser undergrowth.

The terrain modeling method by fitting a line to single scan lines works best when there are only a little undergrowth as usually in Finnish pine forest shown in Fig 1. If there are large differences in the ground or large amount of vegetation or foliage, the ground detector might not found the assumed linear ground level. We had only data from the forests that had only a little undergrowth and the behavior of the terrain detector could not be tested with undergrowth.

Medians of data were used as an estimate for ground level and circle features in the mapping because it is easily computable robust measure of the data. The median was in practice more robust against outliers than average of the data. More complex estimates were not used because the median was giving nearly similar values as the average of the data without outliers. The accuracy of terrain model was not as important as the accuracy of the tree trunk measurements, because terrain model was only used to set reference height for tree diameter measurements.

It is quite challenging to get good reference measurement from the forest to test the tree trunk detection and measurement algorithms. Highly accurate RTK-GPS won't work sufficiently under the tree canopy at the northern regions as the satellites are seen near the horizon behind the tree canopy and all the foliage. The trees are traditionally measured by hand using a diameter at breast height and that information is difficult to combine to measurements of tree profile at different heights. In addition, it is laborious to take reference measurements from the whole forest including all tree trunks with an accurate location and diameter measurements at different heights. We are planning to make an accurate $3 \mathrm{D}$ model of a forest by using Riegl 3D scanner and stitching the data together to get reference model of the forest to compare the accuracy of our algorithms.

Similarly we are building a new version of the rotating 3D laser scanner to have more accurate measurements from the forest. The measurements are more difficult to gather while moving, but when building a measurement system for forest harvester, it should be possible to measure while moving. If the system would require stationary measuring, it would reduce the working efficiency of the forest harvester. All of the algorithms and methods used in this paper are designed for continuous operation and that the high amount of measurement data can be compressed to the mapped features in real time.

\section{CONCLUSIONS}

The paper presents a robust method to extract tree trunks from a noisy 3D point cloud. The geometry of the self-built 3D scanner is used as an advantage in the tree trunk and ground detection. The first data segmentation is done for single scan lines. Returns from adjacent angles are used to detect the edges of tree trunks. Because of the $45^{\circ}$ angle, the ground and different trees can be detected and segmented from a single laser scan using presented methods.

The point cloud data can be buffered and updated in a way that the pose changes, which are accumulated during taking a whole 3D scan, are compensated out from the final 3D point cloud. After the pose compensation, the terrain model and separate trees can be searched and modeled from the $3 \mathrm{D}$ point cloud using the presented $2 \mathrm{D}$ histogram method. The buffered point cloud needs to be collected at least for one second to get $3 \mathrm{D}$ measurements equally from every direction around the vehicle.

In our work, the system is used on a platform that moves while the $3 \mathrm{D}$ scanner is measuring. The measuring and modeling is more challenging as the measuring vehicle is moving. By using inertial measurements and a laser odometry method designed for forest environment to track pose changes during the one second time interval, it is possible to compensate the movement from the 3D point cloud.

The terrain model is used as a reference level for feature extraction and tree trunk modeling. The features can therefore be mapped using a 2D map instead of a more complex 3D map. The idea of using highly refined features in a SLAM process is the key to reduce the calculation complexity and simultaneously acquire trunk diameter measurements at different heights in a forest. 


\section{ACKNOWLEDGEMENTS}

This work was done under the Forestrix-project, which was funded by TEKES, Finnish Funding Agency for Technology and Innovation and participating companies. The work was assisted by two researchers from Aalto University, M. Sc. Mikko Miettinen who assisted the data collection, and M. Sc. Matti Öhman who built the rotating 3D scanner.

\section{REFERENCES}

Aschoff, T., Thies, M. \& Spiecker, H. (2004) "Describing forest stands using terrestrial laser-scanning", International Archives of Photogrammetry, Remote Sensing and Spatial Information Sciences, vol. 35, no. Part B, pp. 237241.

Bailey, T. \& Nebot, E. (2001) "Localisation in large-scale environments", Robotics and Autonomous Systems, vol. 37, no. 4, pp. 261-281.

Coope, I.D. (1993) "Circle fitting by linear and nonlinear least squares", Journal of Optimization Theory and Applications, vol. 76, no. 2, pp. 381-388.

Forsman, P. (2001) "Three-dimensional localization and mapping of static environments by means of mobile perception", Helsinki University of Technology.

Guivant, J. \& Nebot, E. (2001) "Simultaneous localization and map building: Test case for outdoor applications", Australian Centre for Field Robotics, Tech.Rep.

Henning, J.G. \& Radtke, P.J. (2006) "Detailed stem measurements of standing trees from ground-based scanning lidar", Forest Science, vol. 52, no. 1, pp. 67-80.
Hyyti, H. (2009) "Simultaneous localization and forest mapping using 2D and 3D laser scanners", Master's Thesis, Helsinki University of Technology.

Hyyti, H., Öhman, M., Miettinen, M. \& Visala, A. (2009)

"Heuristic Correlation based Laser Odometry Method for Unconstructed Environment", Robotics and Applications ACTA Press.

Jutila, J., Kannas, K. \& Visala, A. (2007) "Tree measurement in forest by 2D laser scanning", Computational Intelligence in Robotics and Automation, 2007. CIRA 2007. International Symposium on IEEE, pp. 491.

Lalonde, J.F. (2006) "Natural terrain classification using three-dimensional ladar data for ground robot mobility", Journal of Field Robotics, vol. 23, no. 10, pp. 839.

McDaniel, M.W., Nishihata, T., Brooks, C.A., Salesses, P. \& Iagnemma, K. (2012) "Terrain classification and identification of tree stems using ground-based LiDAR", Journal of Field Robotics, vol. 29, no. 6, pp. 891-910.

Metla (2007) "Forest Finland in Brief", [Online], Available: http://www.metla.fi/metinfo/tilasto/julkaisut/muut/brief20 07.pdf [24. Aug 2009].

Nüchter, A., Lingemann, K., Hertzberg, J. \& Surmann, H. (2007) "6D SLAM -3D mapping outdoor environments", Journal of Field Robotics, vol. 24, no. 8-9, pp. 699-722.

Weisstein, E.W. (2012) "Least Squares Fitting", [Online], Available: http://mathworld.wolfram.com/ LeastSquaresFitting.html [19. Nov 2012]. 